$\mathrm{ECT}^{*}-99-2$

March 1999

\title{
Effect of a scale-dependent cosmological term on the motion of small test particles in a Schwarzschild background
}

\author{
Giovanni Modanese日 \\ European Centre for Theoretical Studies in Nuclear Physics and Related Areas \\ Villa Tambosi, Strada della Tabarelle 286 \\ I-38050 Villazzano (TN) - Italy \\ and \\ I.N.F.N. - Gruppo Collegato di Trento \\ Dipartimento di Fisica dell'Università \\ I-38050 Povo (TN) - Italy
}

\begin{abstract}
It was recently suggested that the gravitational action could contain a scale-dependent cosmological term, depending on the length or momentum scale characteristic of the processes under consideration. In this work we explore a simple possible consequence of this assumption. We compute the field generated in empty space by a static spherical source (the Schwarzschild metric), using the modified action. The resulting static potential turns out to contain a tiny non-Newtonian component which depends on the size of the test particles. The possible relevance of this small correction for the analysis of the recent Pioneers data [J.D. Anderson et al., Phys. Rev. Lett. 81 (1998) 2858] is briefly discussed. 04.20.-q Classical general relativity.

04.60.-m Quantum gravity.

Key words: General Relativity, Quantum Gravity, Experimental Gravitation
\end{abstract}

\footnotetext{
1e-mail: modanese@science.unitn.it
} 


\section{Introduction}

On the base of the work by Hamber and Williams in Euclidean lattice quantum gravity «1], it was recently suggested that the gravitational action could contain a scale-dependent cosmological term [2]. In terms of quantum field theory this means that the effective value of the cosmological constant would depend on the characteristic length or momentum scale of the processes under consideration. Obviously since quantum gravity is not renormalizable, at least in the pure Einstein formulation, the concept of an effective coupling depending on the scale is not well defined at the perturbative level. A non perturbative analysis is needed, like that of Ref. [1].

The issue of a scale-dependent cosmological term is connected to the well known puzzle of the "global" cosmological constant $\Lambda$. In principle this can be present in the gravitational action already at the classical level and is expected to receive a large contribution from the vacuum fluctuations of the quantum fields; still, its observed value is very close to zero. Several theoretical conjectures were formulated in order to explain this cancellation [3]. The experimental upper limits on a global $\Lambda$ [4] stem from observations at cosmological scale and also from astronomical measurements in the solar system, where a negative $\Lambda$ would correspond to a - never observed - Yukawa range for the gravitational potential of the sun and the planets.

\subsection{The scaling law}

How does the effective value $\Lambda_{\text {eff }}$ of the cosmological constant (i.e., the average value of the scalar curvature evaluated in a certain 4D region) depend on the scale in the quantum lattice theory? It turns out that $\Lambda_{\text {eff }}$ tends to zero when the size $\xi$ of the region goes to infinity, but for any finite value of $\xi$ it is given by the power law

$$
\left(\left|\Lambda_{e f f}\right| G\right)(\xi) \sim(l / \xi)^{\gamma}
$$

where $l$ is the lattice spacing and $\gamma$ is a critical exponent. This scale dependence makes sense as long as we regard $l$ as a small but finite quantity. In fact in lattice quantum gravity, unlike in the usual lattice theories, the lattice spacing not only acts as a regulator, but also represents the minimum physical distance. Thus $l$ cannot be smaller than the Planck length and in the following $l$ will indeed denote $l_{P} \sim 10^{-33} \mathrm{~cm}$. (In this work we employ natural units, such that

$\hbar=c=1$. Since in terms of $\hbar, c$ and $G$ one has $l_{P} \sim \sqrt{G \hbar / c^{3}}$, the scale dependence of the cosmological term can be regarded as a quantum effect.)

Note that being $l$ extremely small, the ratio $l / \xi$ is also very small at any reasonable scale. 
Also note that in order to isolate $\left|\Lambda_{\text {eff }}\right|$ in eq. (1) we must do some hypothesis on the behavior of the effective Newton constant $G$. It is generally believed - and the numerical simulations confirm this to some extent - that the scale dependence of $G$ is quite weak. Thus after extracting $\left|\Lambda_{e f f}\right|$ from the adimensional product $\left|\Lambda_{e f f}\right| G$ one finds that $\left|\Lambda_{e f f}\right|$ varies essentially as $(l / \xi)^{\gamma}$.

The critical exponent $\gamma$ has been numerically estimated in the simulations, but only for small lattices. Some consistency arguments (compare Section 2) allow to conclude that $\gamma$ is slightly larger than 2. The simulations also show that the sign of $\Lambda_{\text {eff }}$ is negative: when the size of the $4 \mathrm{D}$ region, where the average of the effective value of $\Lambda$ is computed, tends to infinity, $\Lambda_{\text {eff }}$ tends to zero from below.

This feature is familiar in numerical quantum gravity on the Regge lattice, where a positive value of the effective $\Lambda$ always corresponds to the unstable, collapsed phase of the system. It is also connected, in the weak field limit, to a property of the linearized theory, where a positive cosmological term corresponds to an imaginary mass for the graviton and thus to unstable solutions.

Although most of the considerations above refer (like the numerical simulations) to the Euclidean field theory, the assumption is made that in the weak field limit this is equivalent to the theory in Minkowski space. However, even if we do not trust this equivalence, we can regard eq. (11) as a reasonable ansatz for the scale dependence.[?

\subsection{Physical implications}

Suppose we accept the possibility of a scale-dependent cosmological term, stronger at short distances than at large distances. The next step is to understand the physical implications of this formal assumption. Which physical processes could be affected by such a term? How can it be taken into account?

In an earlier work we studied whether a local cosmological term could let a graviton decay into collinear gravitons of smaller frequencies. The decay of a massless particle is a quite exotic process, whose amplitude obeys certain general rules [6]; one of these is that decay is possible if the massless field self-interacts with a coupling of positive mass dimension (like $\Lambda$ for the gravitons). However, for any field model comprising a scale-dependent coupling, the separation

\footnotetext{
${ }^{2}$ Besides the numerical simulations, there is a further argument showing that a small negative local cosmological term with the scaling law (1) is necessary for the stability of the gravitational vacuum: it stabilizes the Einstein action with respect to a special set of field configurations, called "zero-modes" [5]. This holds in the Euclidean as well as in the Minkowskian formalism.
} 
of long distance and short distance effects is not trivial. In the case of gravity this problem is even more serious, because while speaking of gravitons as of elementary particles we rely on the concept of Lorentz symmetry; but at the same time we admit that a decay of these particles could be caused by a non vanishing cosmological term, which corresponds classically to a curved background. Therefore it was suggested to consider separately (i) the phase space for the decay products at large distance (referred to flat spacetime), and (ii) a local decay amplitude (where the effective value of $\Lambda$ would appear). This program, however, faces too many technical difficulties and inconsistencies to allow a clear resolution.

This work aims at investigating the possible effects of a scale-dependent cosmological term in a simpler, classical context. In Sections 3 and 4 we study the motion of a body with given finite size in a background gravitational field, under the assumption that this body also "feels" a nonzero local cosmological constant, depending on the body size as in eq. (11). We wonder whether the motion is affected, and to what extent.

There are some paradoxes and physical mistakes which lie in wait for us along this calculation. The first, serious problem is the violation of the equivalence principle. In the presence of purely gravitational forces, any dependence of the center of mass motion of a test body on its size represents such a violation and could be in principle detected by an Eötvös-like experiment. [1 Decisive points are, of course, the magnitude of the violation and its exact dependence on the test body size. This dependence could be so weak to lead to negligible differences in the motion, except for bodies differing in size by several magnitude orders. I

A further crucial problem is a possible violation of the Lorentz symmetry of free motion. In an empty space, how could the motion of a test body be affected by the local $\Lambda$-term without spoiling Lorentz symmetry? Certainly the body cannot "slow down" or "turn" in some direction. Our calculations show that Lorentz symmetry is actually preserved and that the $\Lambda$-term only has an effect in the presence of a non-flat background defining a preferred direction.

In order to fix the ideas we chose a Schwarzschild background metric, i.e., the static spher-

\footnotetext{
${ }^{3}$ According to eq. (11) and the following discussion, the scale dependence of $\Lambda_{\text {eff }}$ is a quantum effect, an "imprint" of the Planck length physics. One could dispute, at this point, how the quantization of a theory intrinsically invariant under diffeomorphisms can lead to results in contrast with the equivalence principle. In fact, as already discussed by Hamber and Williams, the quantum version of General Relativity on the Regge lattice reproduces the complete diffeomorphisms invariance only in the long distance limit.

${ }^{4}$ The experimental data of Ref. [7] are quite inspiring under this respect. Compare our discussion in Section 5.
} 
ically symmetric field generated by a certain mass $M$. We found an expression for the modification of this metric due to the inclusion of local cosmological term in the gravitational action, and studied the motion of a test body in the resulting field. We concluded that the main effect of the cosmological term is just a small unobservable "renormalization" of the product $G M$, independent on the test body size; in addition there is, however, a tiny non-Newtonian force, larger for smaller bodies and depending on the velocity $v(r)$ (but unrelated to the familiar post-Newtonian corrections).

The discussion of this puzzling result is the subject of Section 5.

\section{The critical exponent in the $\Lambda_{e f f}(\xi)$ law}

Before embarking in the main calculation, let us derive in this section an estimate of the exponent $\gamma$ in eq. (11). This is obtained just imposing a phenomenological consistency condition. From eq. (1) we can isolate $\left|\Lambda_{\text {eff }}\right|(\xi)$, obtaining

$$
\left|\Lambda_{e f f}\right|(\xi) \sim l^{-2} \xi^{-\gamma} l^{\gamma}
$$

The range $\rho$ of a Yukawa component in the gravitational potential is proportional to $\left|\Lambda_{e f f}\right|^{-2}$. We thus have (up to a numerical factor of order 1 which is already present in eq. (1))

$$
\rho \sim l^{1-\gamma / 2} \xi^{\gamma / 2}
$$

We see from this equation that if $\gamma$ was exactly equal to 2 , then $\rho=\xi$, i.e. the range of the Yukawa component of the gravitational field observed at the scale $\xi$, would be equal to $\xi$. But we know that such a Yukawa component is never observed, thus we must have $\rho \gg \xi$. For instance, by observing the planetary motion we can set an upper bound on the average curvature of spacetime at a scale of the order of the solar system size.

We therefore assume that $\gamma$ is slightly larger than 2 and define $\alpha=\gamma / 2-1$, where $\alpha$ is a small positive number (of the order of 0.03 or less - see below). In this way, eq. (3) becomes

$$
\rho \sim l^{-\alpha} \xi^{1+\alpha}
$$

and the ratio between $\rho$ and $\xi$ is given by

$$
\frac{\rho}{\xi} \sim\left(\frac{\xi}{l}\right)^{\alpha}
$$


This ratio does not actually need to be very large, because the ratio between a possible Yukawa term $V_{Y}$ in the gravitational potential and the usual Newton term $V_{N}$ reduces to an exponential:

$$
\frac{V_{Y}}{V_{N}} \sim \exp \left(\frac{\xi}{\rho}\right) .
$$

Thus $\rho / \xi$ is the logarithm of the ratio between the upper bound on $V_{Y}$ and $V_{N}$. Taking, for instance, $V_{Y} / V_{N}=10^{-9}$, one finds $\rho / \xi=\ln \left(10^{9}\right) \simeq 21$. The size $\xi$ of the solar system is of the order of $\xi=10^{10} \mathrm{Km}=10^{15} \mathrm{~cm}$, thus the ratio $\xi / l$ in eq. (5) is of the order of $10^{48}$. By requiring $\left(10^{48}\right)^{\alpha}<21$, one finds $\alpha<0.03$ and $2<\gamma<2.03$.

\section{The correction to the Schwarzschild metric}

In this section we compute to lowest order in the weak field approximation the field generated by a pointlike static source, assuming that the field equations contain a cosmological term localized at a given point.

\subsection{Einstein field equations and harmonic gauge propagator}

Let us start by fixing our conventions. The Einstein equations in the absence of a cosmological term are

$$
R_{\mu \nu}(x)-\frac{1}{2} g_{\mu \nu}(x) R(x)=-8 \pi G T_{\mu \nu}(x) .
$$

The trace of this equation is $R(x)=8 \pi G T(x)$, where $T$ is the trace of $T_{\mu \nu}$.

We focus on the case of a weak field $g_{\mu \nu}(x)=\eta_{\mu \nu}+h_{\mu \nu}(x)$; neglecting terms quadratic in $h$, one obtains the usual linearized version of Einstein equations, which in harmonic gauge can be written in the form

$$
K_{\mu \nu \rho \sigma}(x) h^{\rho \sigma}(x)=T_{\mu \nu}(x)
$$

where $K$ is a linear differential operator. Given the source $T_{\mu \nu}$, the solution of this equation is

$$
h_{\mu \nu}(x)=\int d^{4} y P_{\mu \nu \rho \sigma}(x, y) T^{\rho \sigma}(y)
$$

where $P$ is the propagator, that is, the inverse operator of $K$, given by

$$
P_{\mu \nu \rho \sigma}(x, y)=-\frac{2 G}{\pi} \frac{Q_{\mu \nu \rho \sigma}}{(x-y)^{2}-i \varepsilon}
$$

with $Q$ a constant tensor:

$$
Q_{\mu \nu \rho \sigma}=\eta_{\mu \rho} \eta_{\nu \sigma}+\eta_{\mu \sigma} \eta_{\nu \rho}-\eta_{\mu \nu} \eta_{\rho \sigma}
$$


In all these equations it is understood that, in accordance with the linearized approximation, the indices are lowered and raised with the metric $\eta_{\mu \nu}$, which is diagonal and has signature ($1,1,1,1)$. The square of a 4 -vector $x$ is $x^{2}=-\left(x^{0}\right)^{2}+\mathbf{x}^{2}$.

\subsection{Field equations with a pointlike source and a local cosmological term}

In the presence of a cosmological constant $\Lambda$ independent on $x$, eq. (7) takes the form

$$
R_{\mu \nu}(x)-\frac{1}{2} g_{\mu \nu}(x) R(x)+\frac{1}{2} \Lambda g_{\mu \nu}(x)=-8 \pi G T_{\mu \nu}(x) .
$$

If $T_{\mu \nu}=0$, this equation admits a solution with constant scalar curvature $R(x)=2 \Lambda$.

The linearized version of eq. (12) is

$$
-8 \pi G K_{\mu \nu \rho \sigma}(x) h^{\rho \sigma}(x)+\frac{1}{2} \Lambda\left[\eta_{\mu \nu}+h_{\mu \nu}(x)\right]=-8 \pi G T_{\mu \nu}(x) .
$$

We want now to solve this equation in the case when the energy-momentum tensor is that of a pointlike mass placed at the origin of the coordinates, called $T_{\mu \nu}^{S}(x)$, and the effective cosmological constant depends on $x\left(\Lambda \rightarrow \Lambda_{\text {eff }}(x)\right)$. Let us decompose the metric $h_{\mu \nu}(x)$ as follows:

$$
h_{\mu \nu}(x)=h_{\mu \nu}^{S}(x)+\Lambda_{e f f}(x) h_{\mu \nu}^{\Lambda}(x)
$$

where the metric $h^{S}$ satisfies the equation

$$
K_{\mu \nu \rho \sigma}(x) h^{S, \rho \sigma}(x)=T_{\mu \nu}^{S}(x)
$$

and can be therefore regarded as the linearized version, in harmonic gauge, of the Schwarzschild metric. (We shall derive $h^{S}$ in a moment.) By introducing the decomposition (14) into eq. (13) we obtain

$$
\begin{aligned}
& -8 \pi G K_{\mu \nu \rho \sigma}(x)\left[h^{S, \rho \sigma}(x)+\Lambda_{e f f}(x) h^{\Lambda, \rho \sigma}(x)\right]+ \\
& +\frac{1}{2} \Lambda_{e f f}(x)\left[\eta_{\mu \nu}+h_{\mu \nu}^{S}(x)+\Lambda_{e f f}(x) h_{\mu \nu}^{\Lambda}(x)\right]=-8 \pi G T_{\mu \nu}^{S}(x) .
\end{aligned}
$$

Now let us write the function $\Lambda_{e f f}(x)$ in the form $\Lambda_{e f f}(x)=\Lambda_{e f f} f(x)$, where $f(x)$ is an adimensional function vanishing outside a region of width $d$ (size of the test particle) centered at $\mathbf{X}$ (center of mass coordinate of the test particle), and such that $f(\mathbf{X})=1$. In eq. (16) we can single out terms of zeroth order in $\Lambda_{\text {eff }}$, which cancel out due to eq. (15), one term of order 
$\Lambda_{\text {eff }}^{2}$, which can be neglected, and terms of the first order in $\Lambda_{\text {eff }}$, which lead to the following equation for the metric $h^{\Lambda}$ :

$$
-8 \pi G K_{\mu \nu \rho \sigma}(x) f(x) h^{\Lambda, \rho \sigma}(x)+\frac{1}{2} f(x)\left[\eta_{\mu \nu}+h_{\mu \nu}^{S}(x)\right]=0 .
$$

If we know the background metric $h^{S}(x)$, we can find the correction $h^{\Lambda}(x)$ due to the cosmological term in the neighbourhood of $\mathbf{X}$ by applying the operator $P$, inverse of $K$ :

$$
h_{\mu \nu}^{\Lambda}(x)=\frac{1}{8 \pi G f(x)} \int d^{4} y P_{\mu \nu \rho \sigma}(x, y) \frac{1}{2} f(y)\left[\eta^{\rho \sigma}+h^{S, \rho \sigma}(y)\right] .
$$

\subsection{Computation of $h^{S}$}

In order to find $h^{S}(x)$, we replace $T^{S}(x)$ by its explicit expression

$$
T^{S}(x)=M \delta^{3}(\mathbf{x}) \delta_{\mu 0} \delta_{\nu 0}
$$

and integrate eq. (8):

$$
\begin{aligned}
h_{\mu \nu}^{S}(x) & =\int d^{4} y P_{\mu \nu \rho \sigma}(x, y) M \delta^{3}(\mathbf{y}) \delta^{\rho 0} \delta^{\sigma 0}= \\
& =M \int d y^{0} P_{\mu \nu 00}\left(x ; y_{0}, \mathbf{y}=0\right)= \\
& =M \frac{-2 G}{\pi}\left(2 \eta_{\mu 0} \eta_{\nu 0}-\eta_{\mu \nu} \eta_{00}\right) \int d y^{0} \frac{1}{\mathbf{x}^{2}-\left(x^{0}-y^{0}\right)^{2}-i \varepsilon}= \\
& =\frac{2 G M}{|\mathbf{x}|}\left(2 \eta_{\mu 0} \eta_{\nu 0}-\eta_{\mu \nu} \eta_{00}\right) .
\end{aligned}
$$

Then, in conclusion, $h^{S}$ is given by

$$
h_{00}^{S}(\mathbf{x})=\frac{2 G M}{|\mathbf{x}|}\left(\eta_{00}\right)^{2}=\frac{2 G M}{|\mathbf{x}|}
$$

which is correct, since in general in a static field one has $h_{00}=-2 V_{\text {Newtonian }}$; the $i i$ components are the same:

$$
h_{i i}^{S}(\mathbf{x})=\frac{2 G M}{|\mathbf{x}|}\left(-\eta_{i i} \eta_{00}\right)=\frac{2 G M}{|\mathbf{x}|}
$$

\subsection{Computation of $h^{\Lambda}$}

Now we can compute $h^{\Lambda}$. In particular, we are interested into the 00 component, which gives the correction to the static potential felt by the test particle. We evaluate it at the center of mass coordinate $X=\left(X_{0}, \mathbf{X}\right)$ and find

$$
\begin{aligned}
h_{00}^{\Lambda}(X) & =\frac{1}{8 \pi G f(X)} \int d^{4} y P_{00 \rho \sigma}(X, y) \frac{1}{2} f(y)\left[\eta^{\rho \sigma}+h^{S, \rho \sigma}(y)\right] \\
& =-\frac{1}{8 \pi^{2}} \int d^{4} y \frac{2 \eta_{0 \rho} \eta_{0 \sigma}-\eta_{00} \eta_{\rho \sigma}}{(X-y)^{2}-i \varepsilon} f(y)\left[\eta^{\rho \sigma}+h^{S, \rho \sigma}(y)\right] .
\end{aligned}
$$


Before looking at the integral, let us focus on the algebraic part of this expression. The term $\eta^{\rho \sigma}$ in the bracket $\left[\eta^{\rho \sigma}+h^{S, \rho \sigma}(y)\right]$, after multiplication by $\left(2 \eta_{0 \rho} \eta_{0 \sigma}-\eta_{00} \eta_{\rho \sigma}\right)$, gives

$$
\left(2 \eta_{0 \rho} \eta_{0 \sigma}-\eta_{00} \eta_{\rho \sigma}\right) \eta^{\rho \sigma}=2 \eta_{00}-4 \eta_{00}=-2+4=2
$$

In a similar way, for the term $h^{S, \rho \sigma}(y)$ we obtain

$$
\begin{aligned}
\left(2 \eta_{0 \rho} \eta_{0 \sigma}\right. & \left.-\eta_{00} \eta_{\rho \sigma}\right) h^{S, \rho \sigma}(y)= \\
& =2 h_{00}^{S}(y)-\left(\eta_{00}\right)^{2} h_{00}^{S}(y)-\eta_{00} \sum_{i} h_{i i}^{S}(y)= \\
& =\frac{2 G M}{|\mathbf{y}|}(2-1+3)=\frac{8 G M}{|\mathbf{y}|} .
\end{aligned}
$$

Now let us write the function $f$ in the following form

$$
f(y)=1 \text { if }|\mathbf{y}-\mathbf{X}|<d ; \quad f(y)=0 \text { elsewhere }
$$

or

$$
f(y)=\theta(d-|\mathbf{y}-\mathbf{X}|)
$$

Setting for simplicity $X_{0}=0$, since everything is static, we obtain the correction

$$
h_{00}^{\Lambda}(\mathbf{X})=-\frac{1}{8 \pi^{2}} \int d^{4} y \frac{\theta(d-|\mathbf{y}-\mathbf{X}|)}{(\mathbf{y}-\mathbf{X})^{2}-\left(y_{0}\right)^{2}-i \varepsilon}\left(2+\frac{8 G M}{|\mathbf{y}|}\right) .
$$

Introducing the new integration variable $\mathbf{z}=\mathbf{X}-\mathbf{y}$, we obtain

$$
h_{00}^{\Lambda}(\mathbf{X})=-\frac{1}{8 \pi^{2}} \int d y_{0} \int d \mathbf{z} \frac{\theta(d-|\mathbf{z}|)}{\mathbf{z}^{2}-\left(y_{0}\right)^{2}-i \varepsilon}\left(2+\frac{8 G M}{|\mathbf{z}-\mathbf{X}|}\right) .
$$

By integrating the first term in the bracket $(2+8 G M /|\mathbf{z}-\mathbf{X}|)$ one clearly obtains a constant independent from $\mathbf{X}$; this constant is irrelevant in the present case, since it does not give any contribution to the force. (This was expected: in flat space there cannot be any force on the test particle due to the local cosmological term, otherwise Lorentz invariance would be spoiled.) From the second term in the bracket $(2+8 G M /|\mathbf{z}-\mathbf{X}|)$ we obtain

$$
h_{00}^{\Lambda}(\mathbf{X})=-\frac{G M}{\pi^{2}} \int d y_{0} \int d \mathbf{z} \frac{\theta(d-|\mathbf{z}|)}{\mathbf{z}^{2}-\left(y_{0}\right)^{2}-i \varepsilon} \frac{1}{|\mathbf{z}-\mathbf{X}|} .
$$

Since

$$
\int d y_{0} \frac{1}{\mathbf{z}^{2}-\left(y_{0}\right)^{2}-i \varepsilon}=-\frac{\pi}{|\mathbf{z}|}
$$

(compare the calculation of $h^{S}$ ), we finally find

$$
h_{00}^{\Lambda}(\mathbf{X})=\frac{G M}{\pi} \int d \mathbf{z} \frac{\theta(d-|\mathbf{z}|)}{|\mathbf{z}||\mathbf{z}-\mathbf{X}|} .
$$


This integral is well known: apart from the factor $-1 / \pi$, it is the Newtonian potential generated at the point $\mathbf{X}$ by a mass distribution with spherical symmetry centered at the origin, having density $\rho(|\mathbf{z}|)=\theta(d-|\mathbf{z}|) /|\mathbf{z}|$. The integral is convergent, positive, and proportional to $1 /|\mathbf{X}|$; the proportionality constant is a pure number of order 1 multiplied by $d^{2}$.

Therefore

$$
h_{00}^{\Lambda}(\mathbf{X}) \sim \frac{G M d^{2}}{|\mathbf{X}|} .
$$

We recall that the static potential is $V=-h_{00} / 2$. The correction to the Schwarzschild metric is $\Lambda_{e f f}(x) h_{\mu \nu}^{\Lambda}(x)$, which computed at $\mathbf{X}$ gives a correction to the potential of the form

$$
V^{\Lambda}(\mathbf{X}) \sim-\frac{G M \Lambda_{e f f} d^{2}}{|\mathbf{X}|}
$$

where $\Lambda_{\text {eff }}<0$ and " $\sim$ " means that an adimensional constant of order 1 is omitted.

\section{The factor $\left|\Lambda_{e f f}\right| d^{2}$}

Eq. (34) gives the first order correction to the 00 component of the Schwarzschild metric, due to a local cosmological term felt by the test particles. This correction is proportional to the product $\left|\Lambda_{\text {eff }}\right| d^{2}$, where $d$ is the size of the test particles. With the notation of Section 2 , and supposing for the moment that the length scale $\xi$ which fixes the value of $\Lambda_{\text {eff }}$ corresponds simply to $d$, the product $\left|\Lambda_{e f f}\right| d^{2}$ can also be written as $(d / \rho)^{2}$ and is thus of order $(l / d)^{\alpha}$ (eq. (5))

The ratio $(l / d)$ is very small, but since the exponent $\alpha$ is small, too, $(l / d)^{\alpha}$ turns out to be a "reasonable" number also at a macroscopic scale; for instance, if $d=1 \mathrm{~cm}$, then

$(l / d)^{\alpha} \sim\left(10^{-33}\right)^{0.03} \sim 10^{-1}$. Therefore the correction (34) is comparable in magnitude to the Newtonian component $-G M / r$ (we set $|\mathbf{X}| \equiv r$ in the following). In fact, the two contributions to $h_{00}$ are indistinguishable, since they have the same $r$-dependence, or in other words the cosmological correction just seems to lead to a renormalization of the factor $G M$.

\section{$4.1 \xi$ depends on the test particles' velocity}

The simple identification $\xi=d$ is not entirely correct, however. In the following, we show that $\xi$ has a weak dependence on the velocity of the test particle, and thus the $r$-dependence of the cosmological correction is not exactly $1 / r$. We first recall that the value of $\Lambda_{e f f}$ is the result of a spatial average. Now, suppose to follow the motion of the test body for a short time interval 
$\Delta t$; the scale $\xi$ corresponds to the cubic root of the volume swept by the body in this interval, namely

$$
\xi \sim\left(d^{3}+d^{2} v \Delta t\right)^{1 / 3}
$$

We see that $\xi$ depends also on the product $v \Delta t$. For very large bodies (for instance, planets) one can consistently let $\Delta t$ approach zero, so that the $d^{3}$ term in the bracket dominates, and $\xi=d$ with good approximation. For small test bodies, however, the term $d^{2} v \Delta t$ will dominate, unless $\Delta t$ is so small to correspond to an impracticable observation (for instance, if $d=0.1 \mathrm{~m}$ and $v=10^{4} \mathrm{~m} / \mathrm{s}, \Delta t$ should be less than $10^{-5} \mathrm{~s}$ ). In other words, even though we are looking for the contribution of the local cosmological term to the potential $V(r)$ at a given point, for small bodies $\Lambda_{\text {eff }}$ depends on the volume swept in the motion during a short time interval and thus also on the velocity $v$.

\subsection{Dependence of $V^{\Lambda}$ on $v(r)$}

Let us go back to the expression (2) for $\Lambda_{e f f}$, namely $\left|\Lambda_{\text {eff }}\right| \sim \xi^{-2}$ (in this computation we can neglect $\alpha$ as compared to 2 , thus $\gamma \simeq 2$ ). We set $\xi \sim\left(d^{3}+d^{2} v \Delta t\right)^{1 / 3}$ like in eq. (35) and focus on the motion of a small test body, starting from a certain distance $r_{0}$. Disregarding the term $d^{3}$ in comparison to $d^{2} v \Delta t$ we find that during this motion the adimensional ratio $\left|\Lambda_{e f f}(r)\right| /\left|\Lambda_{e f f}\left(r_{0}\right)\right|$ is determined by the ratio of the velocities at the distances $r$ and $r_{0}$ :

$$
\frac{\left|\Lambda_{\text {eff }}(r)\right|}{\left|\Lambda_{\text {eff }}\left(r_{0}\right)\right|}=\left(\frac{v(r)}{v\left(r_{0}\right)}\right)^{-2 / 3} .
$$

Note that $\Delta t$ has dropped from this expression, as one should expect. Inserting it into eq. (34) we obtain:

$$
V^{\Lambda}(r) \sim \frac{G M}{r}\left[d^{2}\left|\Lambda_{e f f}\left(r_{0}\right)\right|\right]\left(\frac{v(r)}{v\left(r_{0}\right)}\right)^{-2 / 3} .
$$

According to the remarks at the beginning of this section, the constant factor $d^{2}\left|\Lambda_{e f f}\left(r_{0}\right)\right|$ is of order $\sim 1$. The $r$-dependence of the factor $\left(v(r) / v\left(r_{0}\right)\right)^{-2 / 3}$ causes a deviation from the pure $1 / r$ behavior. This dependence can be obtained taking into account the total energy conservation. In the present approximation it suffices to consider the usual non relativistic conservation law:

$$
-\frac{m M G}{r}+\frac{1}{2} m v^{2}(r)=-\frac{m M G}{r_{0}}+\frac{1}{2} m v^{2}\left(r_{0}\right)
$$

or, denoting $v^{2}\left(r_{0}\right)$ by $v_{0}^{2}$

$$
\frac{v^{2}(r)}{v_{0}^{2}}=1+\frac{2 M G}{v_{0}^{2}}\left(\frac{r_{0}-r}{r r_{0}}\right)=1+\frac{2 g_{0}}{v_{0}^{2}} \frac{r_{0}}{r}\left(r_{0}-r\right)
$$


where $g_{0}$ is the gravitational acceleration at the distance $r_{0}$. The constant $g_{0} / v_{0}^{2}$ depends on the specific situation. For instance, the field of the sun at distance $r_{0}=1 A U$ is $g_{0} \simeq 10^{-3} \mathrm{~m} / \mathrm{s}^{2}$, and taking as velocity of the test object $v_{0} \sim 10^{4} \mathrm{~m} / \mathrm{s}$ one obtains $g_{0} / v_{0}^{2} \simeq 10^{-11} \mathrm{~m}^{-1}$.

For small displacements from $r_{0}$, the general $r$-dependence in eq. (39) can be expanded in powers of $\left(r-r_{0}\right)$ as follows $\left(b \equiv g_{0} / v_{0}^{2}\right)$

$$
\left(\frac{v(r)}{v\left(r_{0}\right)}\right)^{-2 / 3} \simeq 1+\frac{1}{3} b\left(r-r_{0}\right)+\frac{1}{9} b\left(2 b-\frac{3}{r_{0}}\right)\left(r-r_{0}\right)^{2}+\ldots
$$

After inserting this expression into eq. (37), one finds: (i) a potential 1/r, which corresponds as noticed above to an invisible "renormalization" of the product $M G$; (ii) an irrelevant constant potential; (iii) a potential linear in $r$, namely

$$
V_{\text {Lin }}^{\Lambda}(r) \sim G M\left[d^{2}\left|\Lambda_{e f f}\left(r_{0}\right)\right|\right] b\left(2 b-\frac{3}{r_{0}}\right) r .
$$

Carrying on the expansion (40) to higher orders, one gets potentials growing like $r^{2}, r^{3} \ldots$ This holds, however, only for $r$ very close to $r_{0}$. In general, the expression (39) should be used.

\section{Discussion}

In the previous sections we investigated some consequences of the insertion into Einstein field equations of a local, scale-dependent cosmological term. By this we mean an effective cosmological constant $\Lambda_{\text {eff }}$ (essentially due to the quantum fluctuations of the gravitational field) whose strength depends on the size of the involved spatial region.

For instance, if we consider the motion of a test particle in a given background field, the effective cosmological term felt by the particle depends on the volume swept by the particle in a short time interval during its motion. We computed the contribution of this term in the case of a Schwarzschild background, and found that the potential $V=-h_{00} / 2$ acting on a small test particle, usually of the Newtonian form $V=-G M / r$, is corrected by a small term depending on $r$ as $v^{-2 / 3}(r) / r$, being $v(r)$ the particle velocity.

The notion of a local cosmological term which "escorts" a particle in its motion is consistent with the definition of $\Lambda_{e f f}$ in lattice quantum gravity as the average curvature measured in a region of given size. However, the extrapolation of this notion from lattice theory and its implementation in the field equations could be properly justified only within a yet-to-come full quantum field theory of gravity. The situation reminds certain issues from the pre-QFT years early in this century, when quantum concepts were inserted by hand into classical field theory. 
Our present approach allows, in some sense, to take into account the effect of the quantum gravitational fluctuations on a classical curved background.

The conclusions of our computation, namely the existence of a tiny non-Newtonian component of the field depending on the size of the test particles, can be related to recent experimental results [7] which called into play such a small "unmodeled" gravitational acceleration to explain the fine-detail motion of some space probes (while the planetary motion would not be affected).

However our phenomenological approach does not allow to fix the numerical proportionality constants appearing in eq.s (37), (41). Furthermore, the power expansion in eq. (41) is not suitable for direct comparison with the experimental data. These originate from a complex fitting procedure, applied to the acceleration measured over long paths and based on conventional subtraction of the known parts of the field (with $G M$ obviously constant). If the results of Ref. [7] will be confirmed, it may be worth to consider the inclusion of the potential (37) in these fits.

This work was partially supported by the A.S.P. - Associazione per lo sviluppo scientifico e tecnologico del Piemonte, Torino, Italy.

\section{References}

[1] H.W. Hamber, Nucl. Phys. B 400 (1993) 347; H.W. Hamber and R. Williams, Nucl. Phys. B 435 (1995) 361.

[2] G. Modanese, Phys. Lett. B 348 (1995) 51; Phys. Rev. D 54 (1996) 5002.

[3] S. Weinberg, Rev. Mod. Phys. B 61 (1989) 1; also see J. Greensite, Phys. Lett. B 291 (1992) 405 and references.

[4] For recent data and references see L.A. Kofman, N.Y. Gnedin and N.A. Bahcall, Astrophys. J. 413 (1993) 1; L.M. Krauss and M.S. Turner, Gen. Rel. Grav. 27 (1995) 1137; A.G. Riess et al., Astronom. J. 116 (1998) 1009; S. Perlmutter et al., astro-ph/9812133, Astrophys. J. (to be published).

[5] G. Modanese, Phys. Rev. D 59 (1998) 024004.

[6] G. Fiore and G. Modanese, Nucl. Phys. B 477 (1996) 623. 
[7] J.D. Anderson, P.A. Laing, E.L. Lau, A.S. Liu, M.M. Nieto, S.G. Turyshev, Phys. Rev. Lett. 81 (1998) 2858. 